Document downloaded from:

http://hdl.handle.net/10251/136214

This paper must be cited as:

Pérez-Simbor, S.; Garcia-Pardo, C.; Cardona Marcet, N. (2019). Initial Delay Domain UWB Channel Characterization for In-body Area Networks. IEEE. 1-5.

https://doi.org/10.1109/ISMICT.2019.8743767

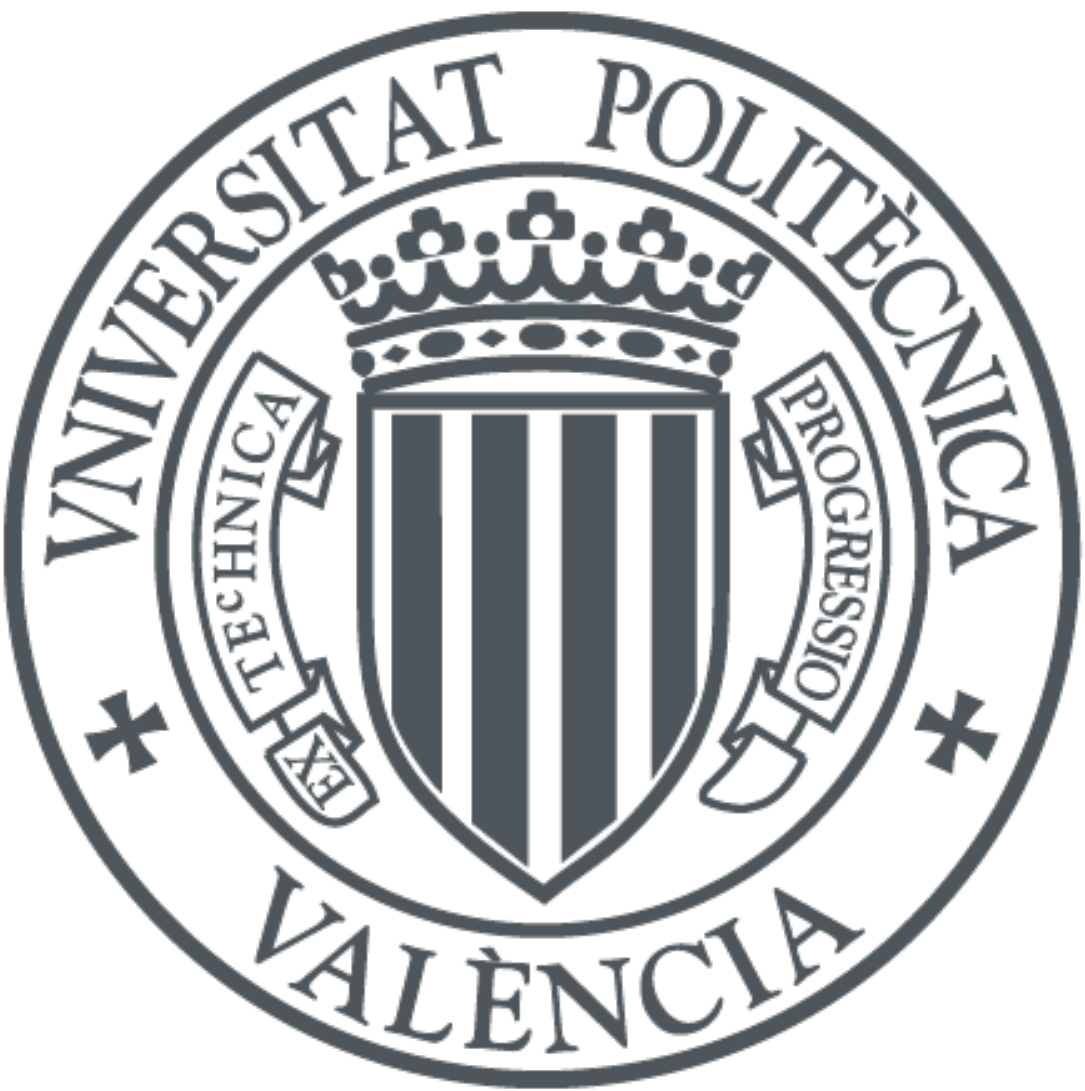

The final publication is available at

https://doi.org/10.1109/ISMICT.2019.8743767

Copyright IEEE

Additional Information 


\title{
Initial Delay Domain UWB Channel Characterization for In-Body Area Networks
}

\author{
Sofia Perez-Simbor, Concepcion Garcia-Pardo, Narcis Cardona \\ Instituto de Telecomunicaciones y aplicaciones Multimedia (Iteam) \\ Universitat Politècnica de València(UPV) \\ València, Spain \\ sopresim@iteam.upv.es
}

\begin{abstract}
Wireless Body Area Networks (WBANs) have increased the attention of the research community for the next generation wireless medical devices. Among others, Wireless Capsule Endoscopy (WCE) aims to transmit better quality images. For this, the Ultra Wideband (UWB) frequency band is becoming a good alternative to currently allocated frequencies for in-body networks, allowing higher data rate and having a low power transmission. Common channel characterization in WBANs are performed in frequency domain, i.e., analyzing the received power as a function of frequency. Nevertheless, indepth studies in delay domain analyzing the impulse response of the channel are barely considered in current literature. In this paper, an initial study in delay domain, i.e., the Power Delay Profile (PDP) characteristics, is performed. Moreover, a comparison between the channel response in frequency and delay domain is performed. This work gives an insightful view of the impulse response of the channel for in-body to on-body communications. For that, an extensive campaign of phantom measurements and software simulations are conducted.
\end{abstract}

Keywords-Wireless Body Area Networks, Ultra Wideband, Wireless Medical Devices

\section{INTRODUCTION}

Wireless medical devices are becoming a valuable way of prevention, detection and treating diseases mainly caused by aging, e.g., cardiopathies, digestive illness, and chronic diseases. For example, the Wireless Capsule Endoscopy (WCE) is an efficient way of detecting polyps, blood or malfunction of the small bowel of the patient. Nevertheless, technology is evolving and for the next generation of wireless medical devices, more data rate and less power consumption are required. For that, the Ultra Wideband (UWB) frequency band allocated from 3.1 to $10.6 \mathrm{GHz}$ is becoming a good alternative to the current Medical Implant Communications System (MICS) band from 402 to 406 $\mathrm{MHz}$ [1]. Moreover, the UWB frequency band allows smaller size of the antennas with the consequent reduction of the capsule (or implanted device) size.

The biggest inconvenient of the UWB frequency band is the high losses that appear in the human body due to the frequency dependence of the human tissues [2]. However, this is not always a problem such as described in [3], where the high losses achieve better security rate for the next generation of leadless pacemakers. Nevertheless, a proper channel characterization is vital for the in-body to on-body communications. There are three methodologies commonly used for the characterization of the channel in in-body area networks: software simulations, experimental measurements in laboratory through phantom and experimental measurements performed in living animals (in vivo). Software simulations are the most feasible to perform because they only require a powerful computer. Unfortunately, they are not as close to reality such as the experiments in laboratory or living animals. Furthermore, the high complexity of the simulations leads to a lot of memory and time resources. Experimental in vivo measurements are the closest to the reality, but the necessity of surgical facilities decrease the feasibility of this kind of measurements. Experimental measurements in phantom are a good trade-off between reality, time, cost and feasibility.

The characteristics of the channel in the delay domain in in-body scenarios are a topic barely explored by the scientific community. Some works are found in [4], [5], nevertheless, these works only consider simulations, not laboratory experiments. Moreover, they study different parameters such as the multipath components that appear in the human body. On the other hand, this manuscript extracts the delay characteristics from the laboratory measurements and then these experimental measurements are compared with software simulations. Moreover, a previous characterization in frequency domain was performed in [6], [7], obtaining a formula for the system loss of the radio budget link for the low UWB frequency band (3.1 to $5.1 \mathrm{GHz}$ ). In previous, works the comparison between software simulations, phantom measurements and in vivo measurements showed a high level of agreement in frequency domain achieving very similar system loss model. In order to continue the characterization of the channel, a delay domain analysis is performed

This paper is structured as follows; section II describes the methodology used for the experimental phantom measurements as well as the characteristics of the designed software simulations. Section III explains the theoretical analysis used in this work. Finally, section IV and V present the results and conclusions respectively.

\section{Methodology AND SetuP}

\section{A. Experimental setup}

The phantom measurements performed in laboratory were performed in a dedicated setup developed for the purpose of in-body to on-body measurements in the UWB frequency band. In this section, a rough description will be given, but further details and a picture of the setup can be found in [6]. The setup consists in a small anechoic chamber of $1 \mathrm{~m}^{3}$, which is coated with aluminum foil and flat absorbers designed to attenuate at least $20 \mathrm{~dB}$ of the incident signal. Moreover, inside the chamber, a robotic 3D arm is placed allowing the movement of one of the antennas with a maximum resolution of $1 \mathrm{~mm}$ in $\mathrm{X}, \mathrm{Y}$ and $\mathrm{Z}$ axis. In addition, a magnetic transmitter that creates a magnetic field is placed inside the anechoic chamber; then two magnetic sensors are attached to the antennas giving the exact spatial position of the sensors, thus, the antennas. Regarding the antennas, two quasi-omnidirectional antennas are used for 
the measurements. Concretely, the on-body antenna is a patch antenna of $4 \mathrm{~cm} \times 4.4 \mathrm{~cm}$ for the length and width [8] and the in-body antenna is a CPW antenna of $2 \mathrm{~cm} \times 2.3 \mathrm{~cm}$ length and width [9]. Finally, the measurements are performed with a vector network analyzer (VNA). For the channel characterization purposes, the forward transmission coefficient $\left(\mathrm{S}_{21}\right)$ is the parameter under interest.

Moreover, the measurements were conducted in a multilayer phantom container, which has a size of $25 \mathrm{~cm} \times$ $25 \mathrm{~cm} \times 25 \mathrm{~cm}$, divided in two layers of $23 \mathrm{~cm} \times 25 \mathrm{~cm} \times 25$ $\mathrm{cm}$ and $2 \mathrm{~cm} \times 25 \mathrm{~cm} \times 25 \mathrm{~cm}$, where two different phantoms, muscle and fat respectively, were poured. These phantoms are accurate phantoms that match with the reference values given in literature [2] for the full bandwidth under interest, i.e., the low UWB frequency band (3.1 to 5.1 $\mathrm{GHz}$ ) [10], [11]. The usage of these accurate phantoms makes the phantom based measurements reliable because of the high similarities with the real gastrointestinal case, where muscle and fat are the primary tissues.

\section{B. Methodology}

The parameters used for the phantom measurements are summarized in the following table [6]:

\begin{tabular}{cl}
\multicolumn{2}{c}{ TABLE 1. VNA PARAMETERS } \\
\hline \hline Resolution points & $\mathrm{N}=3201$ \\
\hline Frequency band & $\mathrm{f}=[3.1,8.5] \mathrm{GHz}$ \\
\hline Frequency Resolution & $\Delta \mathrm{f}=1.875 \mathrm{MHz}$ \\
\hline Resolution Bandwidth & $\mathrm{f}_{\mathrm{if}}=3 \mathrm{kHz}$ \\
\hline Output Power & $\mathrm{P}=8 \mathrm{dBm}$ \\
\hline
\end{tabular}

The measurements were performed for five different receivers located over the fat layer of the container. The receivers (Rxs) were placed forming a cross, where the central $\mathrm{Rx}$ is placed in the middle of the fat-layer and the other Rxs are located $2 \mathrm{~cm}$ up, down, left and right. Regarding the in-body antenna, it is located inside the muscle phantom, where it is moved $1 \mathrm{~cm}$ in $\mathrm{X}, \mathrm{Y}$ and $\mathrm{Z}$, concretely $(12 \mathrm{~cm} \times 11 \mathrm{~cm} \times 2 \mathrm{~cm})$. Fig. 1 shows a sketch of the location of the different sample points. As seen the onbody antenna is placed on the ZY plane. Moreover, it should be remarked that due to mechanical restrictions, the in-body measurements were aligned with the upper receiver (Green Rx in Fig. 1).

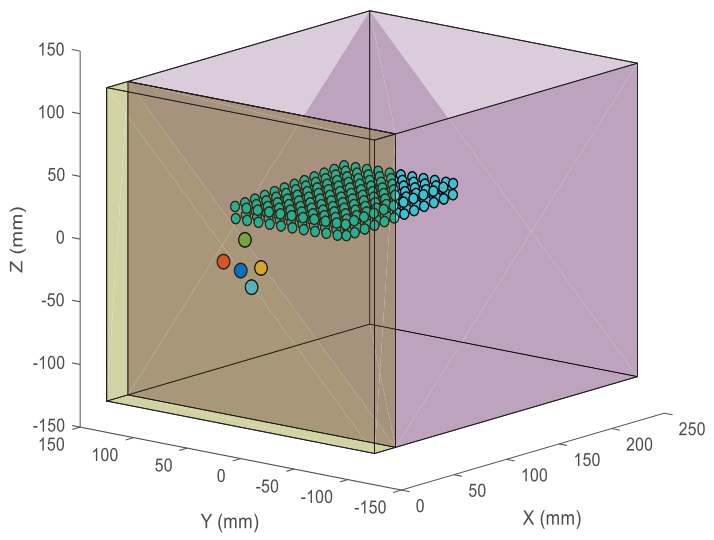

Fig. 1. Spatial sample positions of the phantom measurements [6]

\section{Software simulations}

In order to compare with the phantom measurements, software simulations emulating the scenario under interest were performed with the commercial software $\mathrm{CST}^{\circledR} \mathrm{MWS}^{\circledR}$. The simulations were performed to replicate the conditions of the measurements in the laboratory, and with that purpose, the phantom container, the antennas, and the phantoms were designed. Further information, as well as a picture of the simulation setup, can be found in [6]. In the following table, the most important parameters of the design are described.

\begin{tabular}{cl}
\multicolumn{2}{c}{ TABLE 2. SOFTWARE DESIGN PARAMETERS } \\
\hline \hline Software & CST $^{\circledR} \mathrm{MWS}^{\circledR}$ \\
\hline Container Size & $15 \mathrm{~cm} \times 15 \mathrm{~cm} \times 15 \mathrm{~cm}$ \\
\hline Resolution points & $\mathrm{N}=1601$ \\
\hline Frequency band & $\mathrm{f}=[3.1,5.1] \mathrm{GHz}$ \\
\hline Frequency Resolution & $\Delta \mathrm{f}=1.25 \mathrm{MHz}$ \\
\hline N. of in-body positions & 10 \\
\hline Distance Range & $5.5-9.7 \mathrm{~cm}$ \\
\hline Mesh edge size & {$[0.125-2.611] \mathrm{mm}$} \\
\hline
\end{tabular}

Regarding the methodology used for the simulations, 10 different measurements were performed. First, the antennas were aligned, i.e., they were facing each other in the same $\mathrm{Z}$ and $\mathrm{Y}$ axis (Fig. 1). Then, the in-body antenna was moved along the $\mathrm{X}$-axis in $1 \mathrm{~cm}$ per step. Simulations were performed from $\mathrm{d}=5.5 \mathrm{~cm}$ to $9.5 \mathrm{~cm}$ (x points). Then they were fixed at an $\mathrm{X}$ distance of $7.5 \mathrm{~cm}$ and displaced along $\mathrm{Y}$ axis also $1 \mathrm{~cm}$ per step (6 points), achieving distances between $7.8 \mathrm{~cm}$ to $9.7 \mathrm{~cm}$. In total, ten samples were taken for the comparison between the phantom measurements and the software simulations. It must be remarked that each simulation takes approximately one day of computation, while a set of approximately 500 phantom measurements samples can be taken in one day.

\section{THEORETICAL ANALYSIS}

From measurements and the software simulations, the $\mathrm{S}_{21}$ in frequency domain is obtained, and from it, the channel transfer function is calculated as follows:

$$
H(f)=\left|S_{21}(f)\right| e^{\left(j \phi_{S_{21}}(f)\right)}
$$

Being $S_{21}(f)$ and $\phi_{21}(f)$ the magnitude and phase values of the forward transmission coefficient obtained from the measurements performed with VNA. Thus, the channel transfer function gives the information of the relative received power for the considered bandwidth for the certain number of resolution points in frequency $(\mathrm{N}=3201$ from Table 1). From (1) the system losses (SL), which are the losses due to the antennas and the path loss are computed as follows:

$$
S L(d B)=20 \log _{10}\left(\sum_{i} \frac{\left|H\left(f_{i}\right)\right|}{N}\right)
$$

Nevertheless, this information does not give temporal information such as the characteristics of the path, e.g., the delay of the strongest contribution. In order to observe all this behavior of the channel, in the delay domain, the Inverse Fourier Transform must be done, achieving:

$$
h(\tau)=\operatorname{IFFT}[H(f)]
$$

Now, the obtained values are known as the channel impulse response. It should be noticed that the measurements 
performed are real measurements. Therefore, this is the analysis of a limited channel bandwidth. Nevertheless, it is widely known in the literature that for real measurements the signal in frequency cannot be infinite (achieving then a perfect tap-delay model of the channel). Thus the terminology of the impulse response of the system is accepted even when the delay response are not perfect deltas. Therefore, after the conversion to delay domain in (3)

$$
P D P(d B)=20 \log _{10}(|h(\tau)|)
$$

Being PDP, the Power Delay Profile, which is the relative received power as a function of the delay. With this function, it is possible to see the relative received power of the signal with respect to the delay.

\section{RESULTS}

\section{A. Delay domain analysis}

In this section, a detailed analysis of the direct path as a function of the location of the in-body antenna is performed. Fig. 2 and Fig. 3 show the PDP (from (4)) of the signal for different positions when maintaining $\mathrm{Y}$ and $\mathrm{Z}$ axis constant (Fig. 2) and $\mathrm{X}$ and $\mathrm{Z}$ axis constant (Fig. 3). Moreover, for the sake of simplicity the Rx used to plot the figures is the most aligned one (upper, green Rx in Fig. 1).

In Fig. 2 both antennas are considered aligned, i.e., the angle between them is approximately $0^{\circ}$ either in azimuth and elevation. Therefore, for all the chosen samples the length of the fat layer between antennas is the same for all of them $(2 \mathrm{~cm})$. As seen, from $\mathrm{d}=4.9 \mathrm{~cm}$ to $\mathrm{d}=9.9 \mathrm{~cm}$ there is a visible peak in the measurements, i.e., first and strongest line-of-sight contribution. At further distances, the peak becomes weaker and it is not possible to discern the exact delay for the strongest contribution.

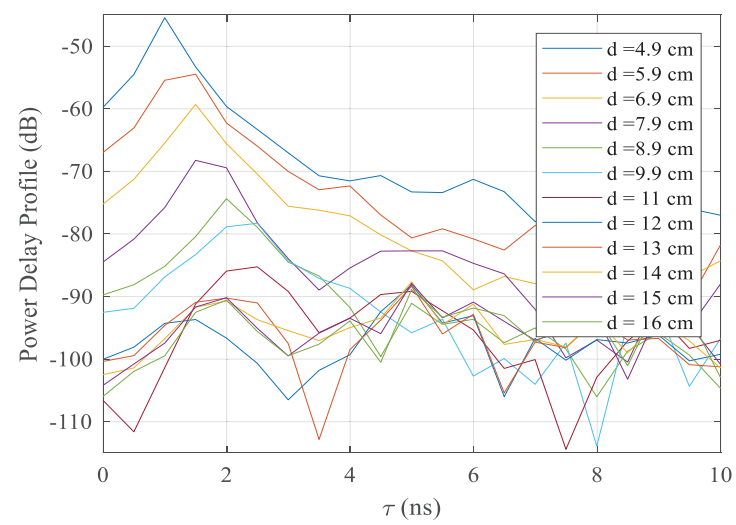

Fig. 2. Delay response for aligned antennas.

These results are consistent with the results obtained in [6], where the $H(f)$ is also showing consistent results until 9.5 $-10 \mathrm{~cm}$ and after that, the signal is below the threshold level, considering the measurements noise. Regarding the power of the strongest contribution, its decay is approximately 8 $\mathrm{dB} / \mathrm{cm}$.

Having an in-depth look in Fig. 2, it is possible to see that for $\mathrm{d}=5.9 \mathrm{~cm}$ and $\mathrm{d}=6.9 \mathrm{~cm}$ the delay corresponding to the first contribution is the same for both measurements approximately $\tau=1.5 \mathrm{~ns}$. Same occurs with $\mathrm{d}=7.9 \mathrm{~cm}$ and $\mathrm{d}$ $=8.9 \mathrm{~cm}$ where the strongest contribution appears at $\tau=2 \mathrm{~ns}$.
This is due to the delay resolution $(\Delta \tau)$ which is the inverse of the considered bandwidth $\Delta \tau=1 / \mathrm{BW}=0.5 \mathrm{~ns}$. As mentioned before, this is due to the finite response of the system that we are measuring. Moreover, continuing with this reasoning, in $\mathrm{d}=5.9 \mathrm{~cm}$ and $\mathrm{d}=7.9 \mathrm{~cm}$ the difference between the strongest and the second contribution is slightly noticeable, it is then, assumable that the strongest contribution lies in between both contributions.

Fig. 3, shows the PDP of different samples, in this case, $\mathrm{X}$ and $\mathrm{Z}$ axis are constant and $\mathrm{Y}$ is the variable. Furthermore, the $\mathrm{Y}$ row chosen is the one with the shortest distance between antennas.

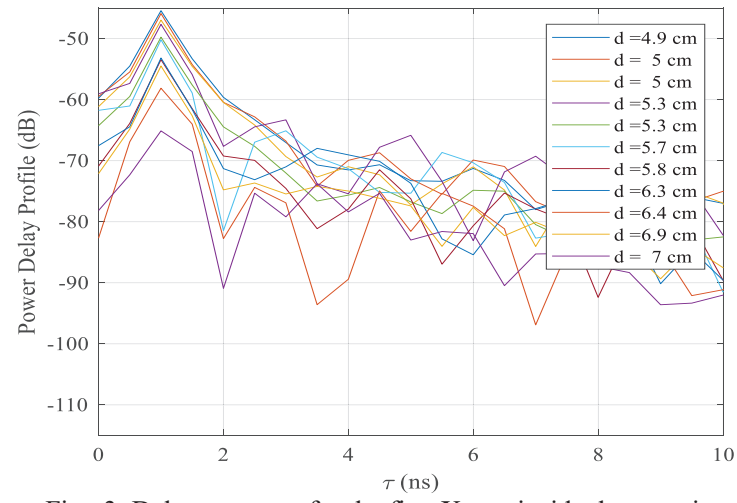

Fig. 3. Delay response for the first $\mathrm{X}$ row inside the containe positions

In Fig. 3 the distances range from $d=4.9 \mathrm{~cm}$ to $d=7 \mathrm{~cm}$. Nevertheless, on contrary than Fig. 2, all the samples plotted have the strongest contributions at $\tau=1$ ns whereas in Fig. 2 for $\mathrm{d}=5.9 \mathrm{~cm}$ and $6.9 \mathrm{~cm}$ the strongest contribution were at $\tau=1.5 \mathrm{~ns}$. These differences can be explained by the length of fat existent between antennas. Now the antennas are displaced in $\mathrm{Y}$-axis, thus the amount of fat between antennas is more than $2 \mathrm{~cm}$, which leads to a faster signal propagation, due to the lower dielectric properties of the fat compared with the muscle. Moreover, there is also a difference in the relative received power of the signal, now the losses per $\mathrm{cm}$ vary between 6 and $10 \mathrm{~dB}$.

Nevertheless, in order to extract a model that can apply to all the measurements in Fig. 5, the value of the maximum contribution of the PDP is plotted as a function of the distance, and considering all the five receivers and sample points until $\mathrm{d}=10 \mathrm{~cm}$

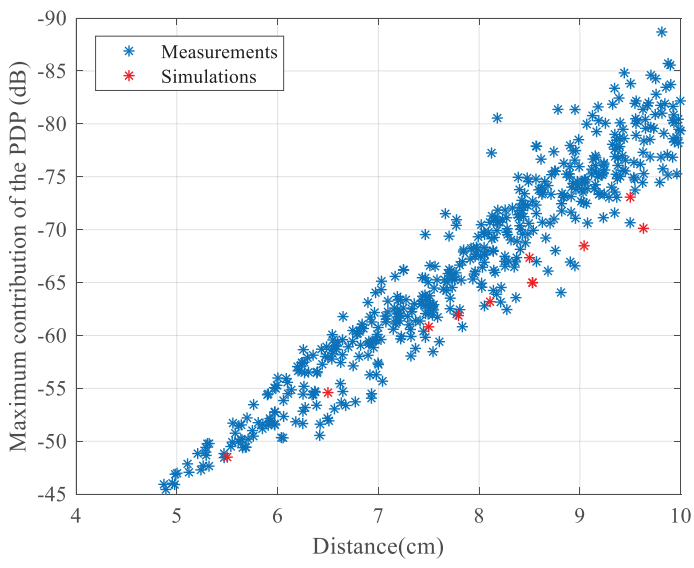

Fig. 4. Maximum contribution of the PDP vs Distance 
Moreover, in Fig. 4 a comparison with software simulations is performed. On the contrary to the measurements in the laboratory were multiple measurements can be performed in one day, the software simulations require an extensive computational time, achieving less amount of simulated in-body points. From Fig. 5, the results obtained with software simulations and phantom measurements have a high level of agreement. As expected, the strongest contributions that appear in software simulations are higher than that deduced from phantom measurements due to the real conditions and the higher misalignment between antennas that measurements experience.

\section{B. Peak of the Power Delay Domain and System Loss Comparison}

In this section, the maximum contribution of the PDP and the System Loss from [6] are compared. From (2) the System Loss is calculated considering the full bandwidth under study $(3.1-5.1 \mathrm{GHz})$ and the result is plotted together with the absolute value of the maximum power contribution in delay domain. From Fig. 5, a visible difference between time and frequency domain is seen. From the measurements, it is possible to see how the system loss in frequency domain is some $\mathrm{dBs}$ lower than the absolute value of the strongest contribution in time domain.

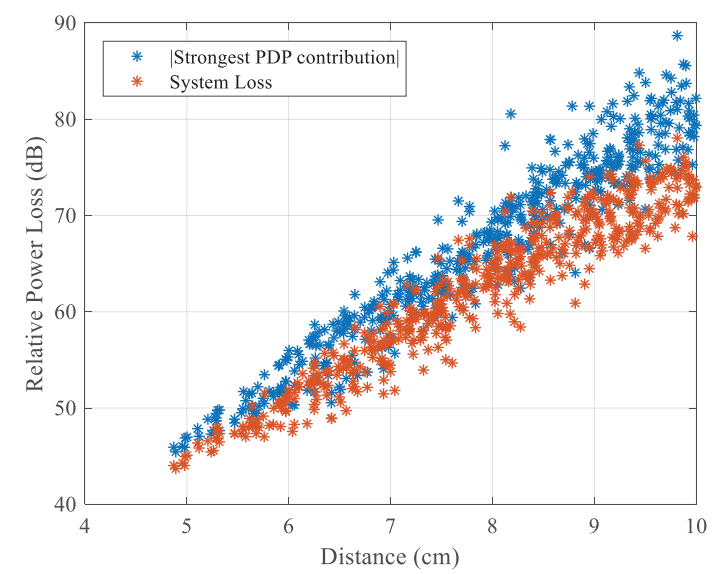

Fig. 5. Comparison between system loss and the strongest PDP contribution

These differences vary from $2 \mathrm{~dB}$ for short distances $(5$ $\mathrm{cm})$ and $7 \mathrm{~dB}$ for longer distances $(10 \mathrm{~cm})$. In Fig. 6 the percentage of this difference is plotted. As seen the difference between the System Loss and the strongest contribution of the PDP suffer a variation from approximately $2 \%$ to $9 \%$. These results let different open possibilities. For example, for low distances, the difference between the strongest PDP contribution and the SL has lower values, e.g., around 3\%. Therefore, it is assumable that almost all the power is received with the first contribution of the signal. Nevertheless, for larger distances, this difference highly increases until almost $9 \%$ of the contribution. Multiple explanations arise from this difference, e.g., multipath components. Another possible explanation can be the delay resolution that the PDP has due to the BW constraint. As already explained, in some cases, such as in Fig. 2 for $\mathrm{d}=5.9 \mathrm{~cm}$ and $7.9 \mathrm{~cm}$, where the maximum contribution of the signal does not seem to have a strong peak but the power is spread in two consecutive bins, thus, a reduction of the maximum received power

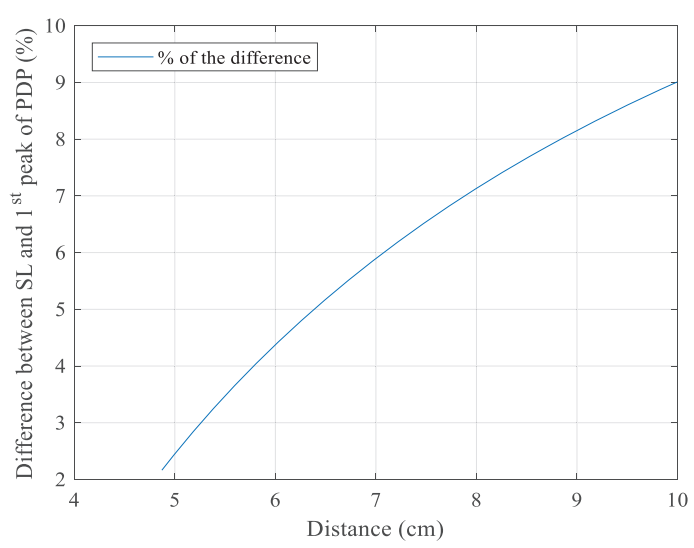

Fig. 6. Difference between the SL and the strongest contribution of the $\mathrm{PDP}$ in $\%$

\section{DISCUSSION AND CONCLUSIONS}

In this paper, an initial analysis in delay domain for the in-body to on-body (IB2OB) scenario is performed with the objective of characterize the channel in delay domain. This delay domain analysis are computed from the channel transfer function, $\mathrm{H}(\mathrm{f})$, which is directly measured in laboratory through a VNA. Then, the Power Delay Profile (PDP) is calculated. It is possible to see how the direct contribution of the measurements is very distinguishable until a distance of 9.5 to $10 \mathrm{~cm}$. Nevertheless, from Fig. 2 and Fig. 3 it is possible to see how the spatial positions and tissues between signals affect the channel. For the same distance but different angle between antennas the delay for the first contribution change.

Besides, a comparison between the previous measurements obtained in [6] in frequency domain and the results obtained in delay domain are performed. The difference between the strongest contribution in delay domain and the system loss vary from $2 \%$ to $9 \%$. The differences obtained between the frequency and the delay domain let open different explanations, such as the existence or not of multipath or the influence of the fat in the measurements. Those are still open issues that need to be researched. Moreover, as further research, a deeper study of the time delay using more human models are required, this means in vivo experiments and software simulations with human models.

\section{ACKNOWLEDGMENT}

This work was supported by the European Union's H2020:MSCA:ITN program for the "Wireless In-body Environment Communication- WiBEC" project under the grant agreement no. 675353. This work was also funded by by the European Union's H2020: MSCA: ITN program for the "mmWave Communications in the Built Environments WaveComBE" project under the grant agreement no. 766231. 


\section{REFERENCES}

[1] C. Garcia-Pardo et al., "Ultrawideband Technology for Medical InBody Sensor Networks: An Overview of the Human Body as a Propagation Medium, Phantoms, and Approaches for Propagation Analysis," IEEE Antennas Propag. Mag., vol. 60, no. 3, pp. 19-33, 2018.

[2] C. Gabriel, "Compilation of the Dielectric Properties of Body Tissues at RF and Microwave Frequencies.," Occupational Environ. Health Directorate, Radiofrequency Radiat. Division, Brooks Air Force Base, TX, USA, Jun. 1996.

[3] M. F. Awan, S. Perez-simbor, C. Garcia-pardo, K. Kansanen, and N. Cardona, "Experimental Phantom-based Security Analysis for Next Generation Leadless Cardiac Pacemaker," Sensors, vol. Special Is, no. Wireless Body Area Networks and Connected Health, pp. 1-21, 2018.

[4] A. Khaleghi, R. Chávez-Santiago, and I. Balasingham, "Ultrawideband statistical propagation channel model for implant sensors in the human chest," IET Microwaves, Antennas Propag., vol. 5, no. 15, p. $1805,2011$.

[5] J. Wang and Q. Wang, "Channel modeling and BER performance of an implant UWB body area link," in 2009 2nd International Symposium on Applied Sciences in Biomedical and Communication Technologies, 2009, pp. 1-4.

[6] S. Perez-Simbor, M. Barbi, C. Garcia-Pardo, S. Castello-Palacios, and
N. Cardona, "Initial UWB in-body channel characterization using a novel multilayer phantom measurement setup," 2018 IEEE Wirel. Commun. Netw. Conf. Work. WCNCW 2018, pp. 384-389, 2018.

[7] S. Perez-simbor, C. Andreu, C. Garcia-pardo, M. Frasson, and N. Cardona, "UWB Path Loss Models for Ingestible Devices," IEEE Trans. Antennas Propag., no. Wireless Healthcare Biotechnology, pp. 1-10, 2019.

[8] C. Tarin, P. Marti, L. Traver, N. Cardona, J. A. Diaz, and E. Antonino, "UWB channel measurements for hand-portable devices: A comparative study," IEEE Int. Symp. Pers. Indoor Mob. Radio Commun. PIMRC, 2007.

[9] C. Andreu, C. Garcia-Pardo, A. Fornes-Leal, M. Cabedo-Fabrés, and N. Cardona, "UWB In-Body Channel Performance by Using a Direct Antenna Designing Procedure," in 11th European Conference on Antennas and Propagation (EUCAP), 2017, p. 5.

[10]N. Cardona, S. Castelló Palacios, A. Fornés Leal, C. García Pardo, and A. Vallés Lluch, "Synthetic Model of Biological Tissues for Evaluating the Wireless Transmission of Electromagnetic Waves," Patent WO/2017/109252.

[11] S. Castelló-palacios, C. Garcia-pardo, A. Fornes-leal, N. Cardona, and A. Vallés-lluch, "Full-Spectrum Phantoms for $\mathrm{cm}$-Wave and Medical Wireless Communications," in European Conference on Antennas and Propagation, EUCAP, 2017, pp. 1-3. 